\title{
Augmentation de la part de femmes et de médecins étrangers
}

\author{
Stefanie Hostettlera, Esther Kraft ${ }^{b}$ \\ a Dr sc. EPFZ, division Données, démographie et qualité (DDQ) de la FMH; ${ }^{b}$ lic. rer. oec., cheffe de la division Données, \\ démographie et qualité (DDQ) de la FMH
}

\begin{abstract}
Alors que la FMH s'engage depuis de nombreuses années pour des données de qualité élevée, la statistique médicale de la FMH livre des analyses importantes permettant d'obtenir une vue d'ensemble de la situation en matière de soins en Suisse. La division Données, démographie et qualité (DDQ) publie tous les ans les chiffres actuels et les tendances concernant la démographie du corps médical.
\end{abstract}

\section{Statistique médicale de la FMH - une enquête systématique depuis 1934}

L'objectif d'une prise en charge médicale adaptée aux besoins avec un niveau de qualité élevé guide les actions des institutions et des professionnels de la santé. En plus de l'impact de facteurs démographiques, économiques et sociaux, la hausse de la prise en charge liée à une population vieillissante influe sur l'évolution de notre système de santé. Par ailleurs, la demande accrue en postes à temps partiel, l'introduction de SwissDRG, le nombre limité de places d'études en médecine et de postes de formation postgraduée ainsi que le gel des admissions mettent notre système de santé face aux défis d'aujourd'hui et de demain, comme la pénurie de médecins dans certaines disciplines [1]. Si le nombre de médecins en exercice augmente chaque année en Suisse, un regard plus nuancé laisse cependant supposer que la capacité de fournir les prestations de santé atteint ses limites car d'une part l'âge moyen et le travail à temps partiel augmentent chez les médecins et d'autre part, la proportion de généralistes diminue. Dans ce contexte, il est primordial de suivre les évolutions actuelles du domaine de la santé, de détecter les problèmes qui se profilent et de les corriger.

Depuis de nombreuses années, la statistique médicale de la FMH constitue un élément d'investigation important pour clarifier la situation en matière de besoin. Chaque année, dans le Bulletin des médecins suisses et sur son site internet, la FMH publie ses analyses concernant les caractéristiques démographiques, les indications sur l'activité professionnelle des médecins et d'autres données du corps médical. Toutes ces données sont notamment indispensables pour la re- cherche sur les soins mais elles offrent également des arguments probants pour les décisions qui relèvent de la politique de la santé et permettent d'échanger les points de vue. Enfin, elles constituent une source d'information importante pour les organisations médicales et toutes les sociétés intéressées qui les utilisent pour répondre à des questions spécifiques.

\section{Bases de données}

La statistique médicale est établie en fin d'année (jour de référence: 31 décembre de l'année concernée). Le premier volet de la statistique de la FMH repose sur les indications de la banque de données de la FMH ( $\mathrm{n}=34348)$ et contient les principales caractéristiques de la démographie médicale telles que l'âge, le sexe, la nationalité et le lieu où le médecin exerce sa profession. Le deuxième volet regroupe les résultats des enquêtes annuelles concernant l'activité professionnelle (taux d'activité, structures des cabinets, etc.) et repose sur les données autodéclarées (échantillon myFMH) par plus de 12000 médecins et recueillies au moyen d'un questionnaire sur le portail des membres myFMH (taux de réponse: 42,3\%).

\section{Représentativité de l'échantillon myFMH}

Différentes caractéristiques (sexe, secteur, spécialisation) de la population statistique (tous les médecins) et de l'échantillon myFMH ont été comparées entre elles

Vous trouverez de plus amples informations sur la statistique médicale de la FMH, la statistique des membres et les principaux indicateurs sur le site internet de la FMH (www.fmh.ch $\rightarrow$ Services $\rightarrow$ Statistique). La division DDQ se tient volontiers à votre disposition pour tout complément d'information: ddq[at]fmh.ch / 0313591111. 
dans le but de vérifier si les conclusions tirées des données de l'échantillon myFMH ( $n=12364)$ pouvaient être appliquées à l'ensemble de la population des médecins en exercice en Suisse. La fréquence de ces caractéristiques dans l'échantillon est comparable à celle de l'ensemble de tous les médecins (différence $<10 \%)$. Seuls le secteur hospitalier et les femmes du secteur hospitalier sont sous-représentés dans l'échantillon. Pour minimiser ces biais, les chiffres par secteur et par sexe ont été séparés ou pondérés en conséquence.

Dans les études précédentes, la comparaison entre les données autodéclarées concernant le taux d'activité et les prestations TARMED facturées individuellement ou les minutages saisis confirme la pertinence de l'analyse [2-4].

\section{Corps médical - les médecins hommes (encore) majoritaires}

Le nombre des médecins en exercice a continué d'augmenter (cf. fig. 1). En 2014, 34348 médecins (13589 femmes, 20759 hommes) exerçaient en Suisse, soit 1106 de plus que l'année précédente. La part d'hommes $(60,4 \%)$ est encore supérieure à celle de leurs consœurs (39,6\%). En revanche, comparée à celle des hommes, la part des femmes n'a cessé d'augmenter ces dernières années. La hausse de la part des femmes atteint une moyenne annuelle de 4,8\% depuis 1990, alors qu'elle ne dépasse pas 1,2\% chez les hommes. L'évolution observée depuis plusieurs années du rapport hommes-femmes chez les étudiants en médecine et les médecins en formation postgraduée montre également que la part de médecins femmes en exercice va continuer d'augmenter ces prochaines années. En médecine humaine, le nombre de diplômés de sexe féminin est majoritaire depuis déjà dix ans et atteint $61,9 \%$ (femmes 518, hommes 318) [5]. La proportion de femmes est de $54,2 \%$ pour les octrois de titres fédéraux de spécialiste reconnus (1501 au total).

En comparaison internationale, la densité médicale en Suisse de 4,1 médecins par 1000 habitants* est légèrement supérieure à la moyenne (2013: 3,7 médecins par 1000 habitants) [6]. Parmi les cantons suisses, Bâle-

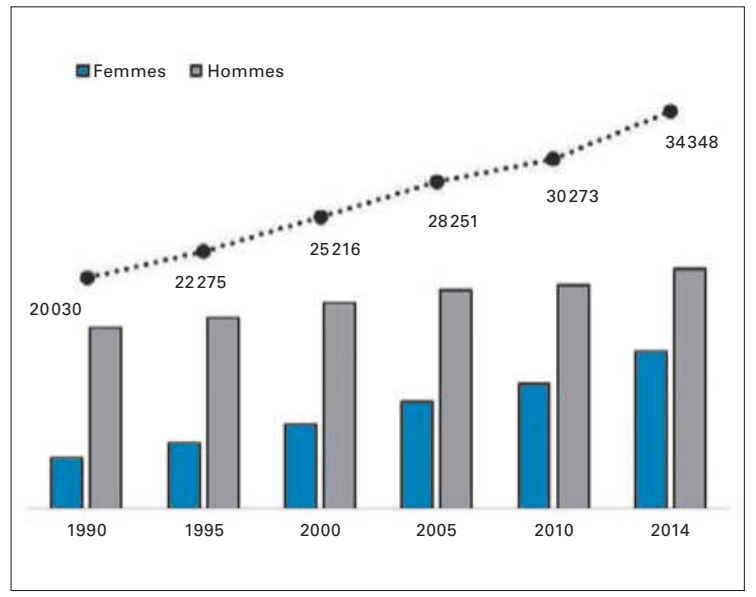

Figure 1: Nombre de médecins d'après l'année et le sexe 1990-2014.

Ville détient la densité la plus forte (avec 9,7 médecins par 1000 habitants), suivi de Genève $(6,2)$ et de Zurich $(4,9)$. Le canton d'Uri (1,6 médecin par 1000 habitants) détient la densité la plus basse, suivi d'Appenzell R. I. $(1,6)$ et Nidwald $(2,2)$.

\section{Lieu de travail - secteur ambulatoire, hospitalier et autre}

La majorité des médecins $(51,8 \%)$ pratiquent leur activité principale dans le secteur ambulatoire (cf. tab. 1) alors que $46,5 \%$ d'entre eux exercent dans le domaine hospitalier et 1,7\% dans un autre secteur (assurances, associations). Par rapport à l'année précédente, le nombre de médecins a augmenté de $1,4 \%$ dans le secteur ambulatoire et de 5,6\% dans le domaine hospitalier. Le nombre de médecins actifs dans un autre domaine (par ex. administration, assurances) a également augmenté (o,4\%). La fig. 2 indique nettement qu'avec l'âge, les médecins exercent davantage dans le secteur ambulatoire.

La part de femmes est plus importante dans le domaine hospitalier $(44,5 \%)$ que dans le secteur ambulatoire (35,5\%). En Suisse, les médecins exercent principalement dans un seul secteur (89,5\%), mais une petite partie d'entre eux pratique dans plus d'un secteur (10,4\%). Parmi ceux-ci, la majorité $(82,5 \%)$ exercent une activité principale dans le secteur ambulatoire tout en occupant simultanément un autre

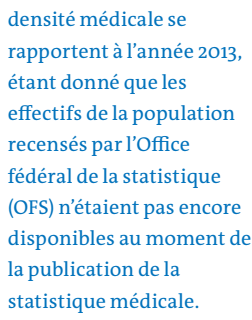

Tableau 1: Aperçu du nombre de médecins en exercice par secteur (activité principale) en 2014.

\begin{tabular}{|c|c|c|c|c|c|c|}
\hline \multirow[b]{2}{*}{ Secteur ambulatoire } & \multicolumn{2}{|c|}{ Femmes } & \multicolumn{2}{|c|}{ Hommes } & \multicolumn{2}{|l|}{ Total } \\
\hline & 6313 & $35,5 \%$ & 11491 & $64,5 \%$ & 17804 & $51,8 \%$ \\
\hline Secteur hospitalier & 7105 & $44,5 \%$ & 8876 & $55,5 \%$ & 15981 & $46,5 \%$ \\
\hline Total & 13589 & $39,6 \%$ & 20759 & $60,4 \%$ & 34348 & $100,0 \%$ \\
\hline
\end{tabular}




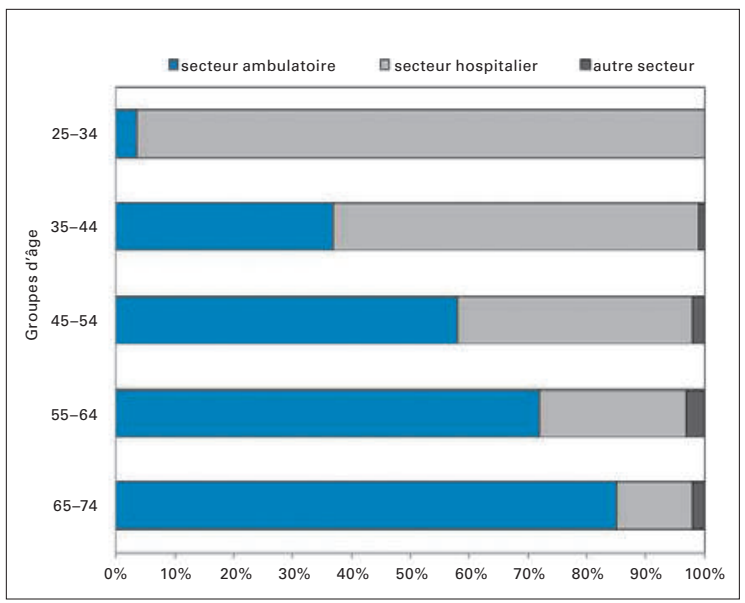

Figure 2: Lieu de travail en fonction de l'âge en 2014.

poste dans le domaine hospitalier, notamment en tant que médecins agréés.

\section{Pyramide des âges - les femmes de moins de 35 ans en supériorité numérique}

En augmentation depuis des années, la moyenne d'âge des médecins en Suisse se situe aujourd'hui à 48,9 ans (cf. tab. 2). Elle était de 45,8 il y a dix ans. Un médecin du secteur ambulatoire a en moyenne 54 ans, tandis que son collègue du secteur hospitalier a 42,9 ans. Cette différence d'âge entre le secteur ambulatoire et le domaine hospitalier s'explique par le fait que la formation postgraduée des assistants se déroule essentiellement en milieu hospitalier. En 2014, les médecins avaient 37 ans en moyenne $(36,5$ pour les femmes, 37,7 pour les hommes) au moment de l'obtention de leur titre de spécialiste, autrement dit au terme de leur formation postgraduée.
La fig. 3 montre qu'en dessous de 35 ans, le nombre de femmes médecins est nettement majoritaire. A partir de 40 ans, le nombre d'hommes en exercice dépasse actuellement celui des femmes. Mais la hausse de la proportion de femmes chez les étudiants et chez les jeunes médecins devrait se refléter dans les différentes tranches d'âge au cours des prochaines années.

\section{Spécialisation - Médecine interne générale en tête}

Le corps médical compte 37,4\% de généralistes (médecine interne générale, pédiatrie et médecin praticien) et $62,6 \%$ de spécialistes. La spécialisation la plus représentée est la médecine interne générale $(23,5 \%)$, suivie de la psychiatrie et psychothérapie (10,3\%), de la gynécologie et obstétrique $(4,8 \%)$, de la pédiatrie $(4,8 \%)$ et de l'anesthésiologie $(4,2 \%)$.

On trouve le plus grand nombre de femmes médecins en psychiatrie et psychothérapie d'enfants et d'adolescents $(62,9 \%)$, en pédiatrie $(57,4 \%)$ et en gynécologie et obstétrique (56,6\%). Le nombre d'hommes est supérieur à celui des femmes dans les spécialisations chirurgicales (chirurgie cardiaque et vasculaire thoracique $92 \%$, chirurgie orthopédique $91,5 \%$, neurochirurgie $86,1 \%$, chirurgie $82,3 \%$ ).

Tableau 2: Age moyen du corps médical en 2014.

\begin{tabular}{llll} 
& Femmes & Hommes & Tous \\
\hline Secteur ambulatoire & 50,9 & 55,7 & 54,0 \\
\hline Secteur hospitalier & 39,6 & 45,5 & 42,9 \\
\hline Tous les secteurs & 45,0 & 51,4 & 48,9
\end{tabular}

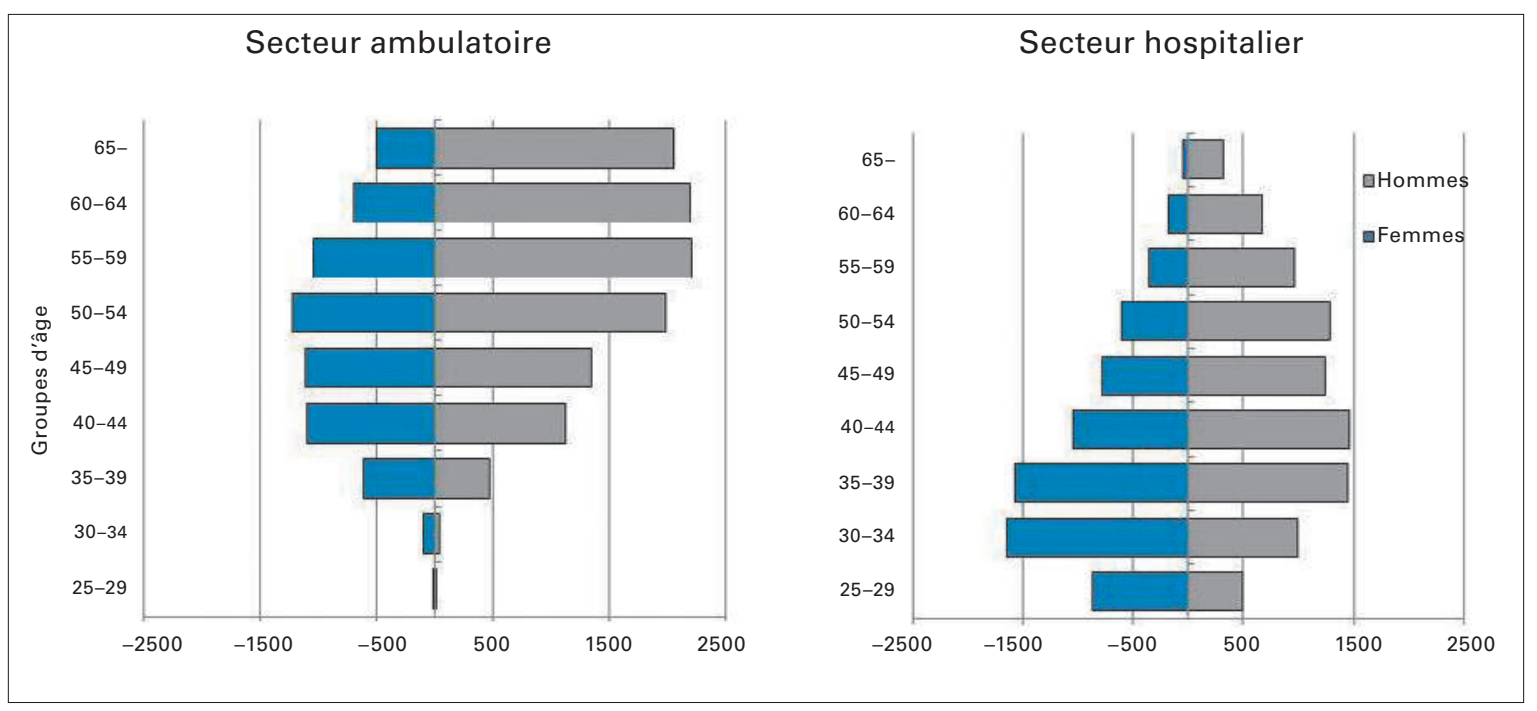

Figure 3: Pyramide des âges et répartition sexo-spécifique des médecins en exercice par secteur en 2014. 
Aujourd'hui, 4571 médecins, dont 1168 femmes, sont porteurs de plusieurs titres. Le titre de spécialiste le plus répandu parmi les porteurs de plusieurs titres est celui de la médecine interne générale (3277). Les porteurs de plusieurs titres exercent principalement en cardiologie (545), en médecine intensive (493) et en rhumatologie (383).

\section{Fonction - baisse du taux de femmes suivant le niveau hiérarchique}

Parmi les 17804 médecins en exercice dans le secteur ambulatoire, 83,6\% sont propriétaires ou propriétaires associés de leur cabinet médical. $4 \%$ des médecins sont engagés en tant qu'assistant ou spécialiste au cabinet

Tableau 3: Aperçu du nombre de médecins par spécialisation (activité principale) en 2014.

\begin{tabular}{|c|c|c|c|}
\hline & Femmes & Hommes & Total \\
\hline Allergologie/Immunologie clinique & 50 & 99 & 149 \\
\hline Anesthésiologie & 591 & 838 & 1429 \\
\hline Cardiologie & 105 & 570 & 675 \\
\hline Chirurgie & 204 & 948 & 1152 \\
\hline Chirurgie orale et maxillo-faciale & 7 & 80 & 87 \\
\hline Chirurgie orthopédique et traumatologie de l'appareil locomoteur & 85 & 920 & 1005 \\
\hline Chirurgie pédiatrique & 27 & 49 & 76 \\
\hline Chirurgie plastique & 45 & 134 & 179 \\
\hline Dermatologie et vénéréologie & 238 & 268 & 506 \\
\hline Gynécologie et obstétrique & 943 & 722 & 1665 \\
\hline Hématologie & 51 & 115 & 166 \\
\hline Infectiologie & 51 & 105 & 156 \\
\hline Médecin praticien & 611 & 659 & 1270 \\
\hline Médecine du travail & 40 & 72 & 112 \\
\hline Médecine intensive & 158 & 355 & 513 \\
\hline Médecine interne générale & 2844 & 5219 & 8063 \\
\hline Médecine légale & 19 & 31 & 50 \\
\hline Médecine nucléaire & 12 & 57 & 69 \\
\hline Neuropathologie & 2 & 13 & 15 \\
\hline Oncologie médicale & 107 & 200 & 307 \\
\hline Ophtalmologie & 379 & 579 & 958 \\
\hline ORL & 118 & 353 & 471 \\
\hline Pas de réponse & 2911 & 2116 & 5027 \\
\hline Pathologie & 115 & 123 & 238 \\
\hline Pédiatrie & 945 & 701 & 1646 \\
\hline Pharmacologie et toxicologie cliniques & 17 & 25 & 42 \\
\hline Pneumologie & 49 & 229 & 278 \\
\hline Prévention et santé publique. & 37 & 41 & 78 \\
\hline Psychiatrie et psychothérapie & 1471 & 2050 & 3521 \\
\hline Psychiatrie et psychothérapie d'enfants et d'adolescents & 392 & 231 & 623 \\
\hline Radiologie & 213 & 584 & 797 \\
\hline Radio-oncologie / Radiothérapie & 40 & 68 & 108 \\
\hline Rhumatologie & 116 & 331 & 447 \\
\hline Urologie & 32 & 272 & 304 \\
\hline Total & 13589 & 20759 & 34348 \\
\hline
\end{tabular}


médical. Le reste des médecins de ce secteur exerce une autre fonction. Les médecins en formation postgraduée (50\% environ) représentent le groupe le plus important du secteur hospitalier. Comme on peut s'y attendre, le nombre de médecins tend à diminuer plus le degré hiérarchique est élevé. On compte par ex. 19,5\% de chefs de clinique, 12,3\% de médecins adjoints et 9,7\% de médecins-chefs. Le nombre de femmes prédomine uniquement chez les médecins-assistants $(57,4 \%)$ et ne fait que diminuer ensuite pour atteindre $43,7 \%$ chez les chefs de clinique, $22,4 \%$ chez les médecins adjoints et seulement 10,9\% chez les médecinschefs.

\section{Les médecins diplômés étrangers viennent principalement d'Allemagne}

Pratiquement un tiers (30,5\%) des médecins en Suisse (10 478) viennent de l'étranger ou plus exactement sont titulaires d'un diplôme étranger en médecine humaine (fig. 4). Parmi eux, 56,2\% exercent dans le domaine hospitalier, $41,7 \%$ dans le secteur ambulatoire et $2,1 \%$ dans un autre domaine. La proportion de médecins étrangers diffère cependant d'une région à l'autre (cf. fig. 4), de 24,1\% dans la région Arc lémanique/Valais à $46,3 \%$ au Tessin. La majorité des médecins étrangers viennent d'Allemagne $(17,4 \%)$, Italie $(2,4 \%)$, France $(1,7 \%)$ ou Autriche $(1,7 \%)$. En comparaison avec l'année précédente, le nombre de médecins en exercice au bénéfice d'un diplôme étranger a
Tableau 4: Taux d'occupation moyen par secteur et par sexe (en demi-journées hebdomadaires) en 2014 ( $n=7295$ ).

\begin{tabular}{llcl} 
& Femmes & Hommes & Total \\
\hline Secteur ambulatoire & 6,8 & 8,9 & 8,2 \\
\hline Secteur hospitalier & 8,8 & 10,1 & 9,4 \\
\hline Autre secteur & 7,2 & 8,9 & 8,3 \\
\hline Total & 7,4 & 9,3 & 8,9 \\
\hline
\end{tabular}

progressé de $1,4 \%$ pour atteindre $30,5 \%$. Avec $44 \%$, la part des femmes est légèrement inférieure à celle des hommes (56\%).

Parmi les 1501 titres de spécialiste décernés en 2014, $44 \%$ l'ont été à des médecins au bénéfice d'un diplôme de médecin étranger.

\section{Taux d'occupation - proportion de temps partiel}

Les indications concernant le taux d'occupation, et toutes les évaluations figurant dans ce second volet, se fondent sur les données autodéclarées par les médecins au moyen d'un questionnaire sur le portail des membres myFMH (échantillon myFMH).

En 2014, les médecins ont travaillé en moyenne 8,9 demi-journées (cf. tab. 4) par semaine. Dans le secteur ambulatoire, le taux d'activité moyen était de 8,2 demi-journées, soit légèrement au-delà de deux demi-journées de moins que dans le secteur hospitalier (9,4 demi-journées). Le taux d'occupation moyen

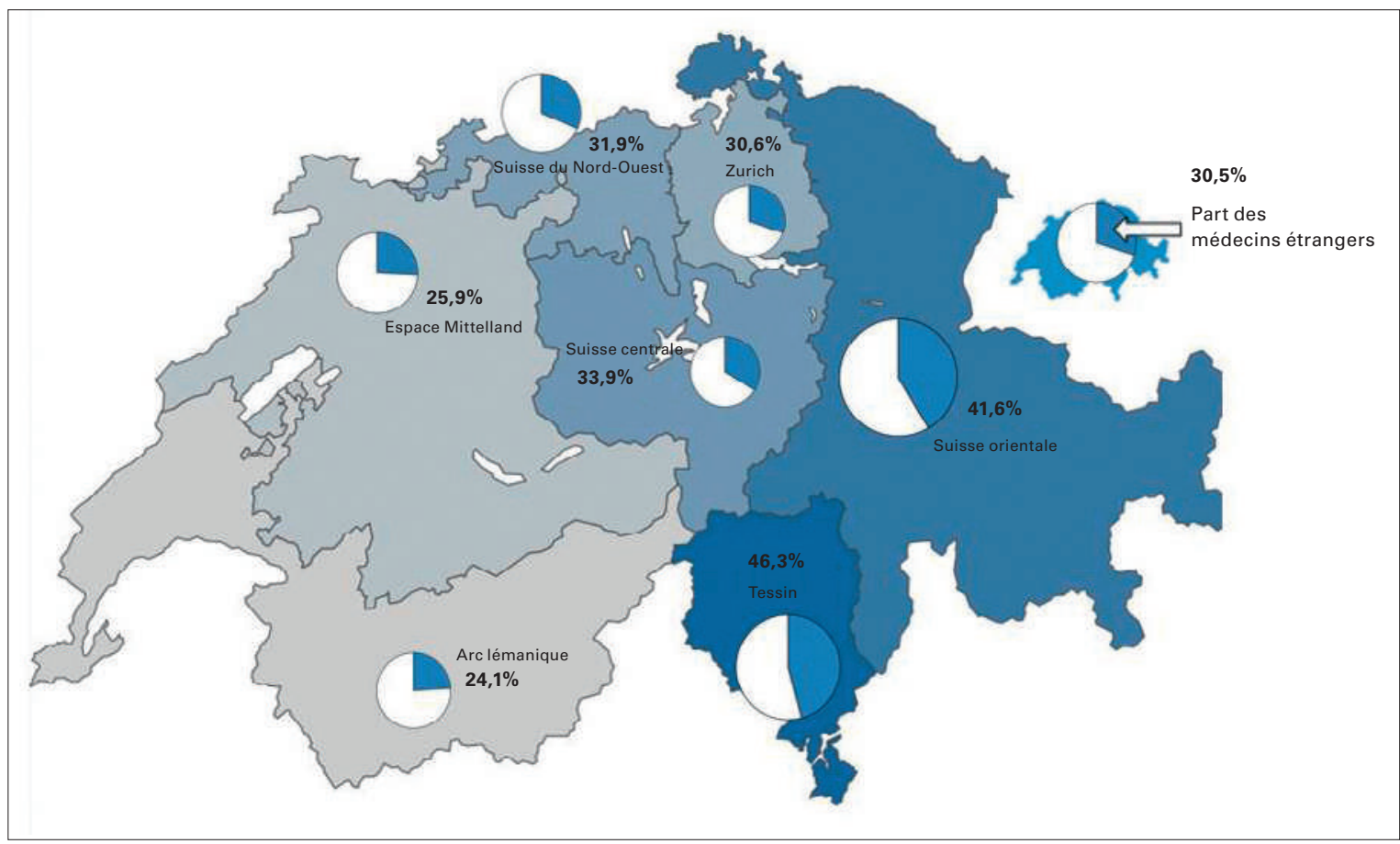

Figure 4: Part de médecins étrangers par région en 2014. 


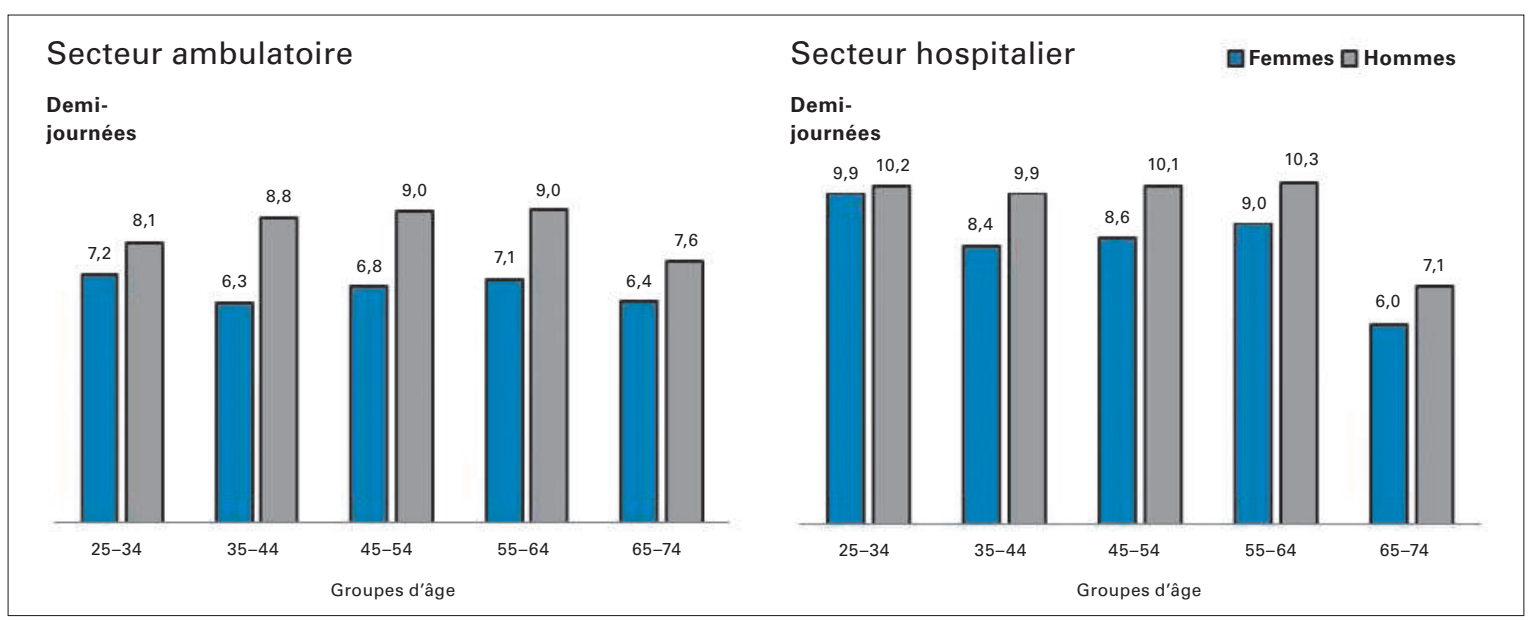

Figure 5: Taux d'occupation par catégorie d'âge et par secteur en $2014(n=7295)$.

des femmes médecins (en ambulatoire: 6,8; en hospitalier: 8,8 demi-journées) est inférieur à celui de leurs collègues masculins (en ambulatoire: 8,9; en hospitalier: 10,1 demi-journées). Depuis 2009, le taux d'occupation moyen a diminué de près de $4 \%$ tandis qu'aucun changement n'a été observé dans le domaine hospitalier.

S'agissant des taux d'occupation, la fig. 5 révèle une différence sexo-spécifique. Dans le secteur ambulatoire, les femmes médecins préfèrent de plus en plus un temps partiel (6o à $80 \%$ ) tandis que le travail à temps partiel est nettement moins répandu dans le domaine hospitalier. Cela est probablement lié au fait que la formation postgraduée des médecins a principalement lieu en milieu hospitalier et que pendant cette période professionnelle, il est plus rare de réduire son temps de travail.

\section{Structures de cabinets et réseaux de médecins}

Dans le secteur ambulatoire, les médecins exercent principalement en cabinet individuel (57,2\%). Depuis 2008 , ce taux a diminué de $5 \%$ environ. La moitié des femmes environ $(48,5 \%)$ exercent dans un cabinet in-

\section{Statistique médicale de la FMH: votre collaboration est essentielle}

Pour réunir des données de bonne qualité, la FMH a besoin du soutien de tous ses membres. Quelques minutes suffisent pour remplir et vérifier le questionnaire sur votre activité professionnelle. Aidez-nous à rendre la statistique médicale de la $\mathrm{FMH}$ encore plus pertinente en vous enregistrant et en vous connectant à $\mathrm{myFMH}$, le portail internet des membres. Vous y trouverez le questionnaire sur la page d'accueil. Nous sommes à votre disposition pour toute question par courriel à myfmh[at]fmh.ch ou par téléphone au 0313591259. dividuel, et $51,5 \%$ dans un cabinet double ou un cabinet de groupe. Chez les hommes, la part de médecins dans un cabinet individuel est légèrement plus élevée à $61,6 \%$. Enfin, un cabinet de groupe compte en moyenne 4 médecins.

Selon les données autodéclarées, 48,9\% des médecins exerçant dans le domaine ambulatoire sont affiliés à un réseau de médecins. Alors que 70,9\% d'hommes médecins sont dans un réseau, la part de leurs collègues féminins est nettement inférieure à $32,8 \%$.

\section{Importance des données et perspective}

La statistique médicale de la FMH rappelle que près d'un tiers des médecins en exercice ( $44 \%$ pour les médecins-assistants) viennent de l'étranger. Faut-il en conclure que le haut niveau de prise en charge ne peut être maintenu dans notre pays que grâce aux médecins formés à l'étranger? Il est urgent que des mesures politiques soient prises pour couvrir le besoin en praticiens avec des médecins formés en Suisse; d'une part, par solidarité pour les pays d'où viennent les médecins étrangers; d'autre part, parce que la possible arrivée de médecins étrangers risque d'être freinée par l'obstacle de la langue. Par ailleurs, certains pays, dont notamment l'Allemagne, réagissent au vaste flux migratoire de leurs médecins vers l'étranger et prennent des mesures pour augmenter ou rappeler l'attractivité des conditions de travail sur leur territoire.

Les différences régionales en matière de densité médicale, l'augmentation de la part des femmes médecins et la plus forte demande en travail à temps partiel qui en découle exigent de créer urgemment de nouveaux modèles de travail. Pour cela, il est décisif que les fournisseurs de prestations procèdent de manière coordonnée et contraignante et qu'ils collaborent au sein d'une même discipline. Dans ce contexte, l'interdis- 
ciplinarité et l'interprofessionnalité sont centrales. Eviter les doublons et les traitements inutiles et inefficaces, améliorer les processus thérapeutiques et utiliser le potentiel existant en vue d'augmenter la qualité des traitements - tous ces points doivent être des principes visant à guider les avancées à venir du système de santé, comme le stipule la stratégie Santé 2020 [7]. La généralisation du dossier électronique du patient, des itinéraires de patients obligatoires et l'encouragement des compétences de santé des patients constituent des facteurs de succès importants.

Le système de santé est un système complexe influencé par de nombreux facteurs. Il est indispensable de disposer de données et de les analyser pour expliquer l'effet et l'efficacité des mesures prises ou à prendre, et pour rester transparent. La statistique médicale de la FMH apporte une contribution décisive pour identifier les besoins et pour répondre à d'autres questions importantes. Tout ceci serait impossible sans la collaboration de nos membres. La FMH saisit donc cette occasion pour vous remercier de votre engagement tout en espérant qu'elle pourra continuer à compter sur votre participation active à l'avenir.

\section{La statistique de la FMH d'un coup d'œil}

Les chiffres intéressants de la statistique médicale 2014 de la FMH sont présentés de manière illustrée et compacte sous forme de poster (en français ou en allemand). Plié, ce dernier a la dimension d'une carte postale: il a donc le format idéal pour être emmené. Nous vous serions reconnaissants si le poster de la statistique médicale de la FMH pouvait trouver sa place dans vos salles d'attente, au cabinet ou à l'hôpital, ou être distribué lors de sessions de formation postgraduée. Nous sommes à votre disposition pour vous transmettre gratuitement le nombre souhaité de posters. Pour cela, il vous suffit de remplir le formulaire de commande sur le site de la FMH (www.fmh.ch $\rightarrow$ Services $\rightarrow$ Statistique $\rightarrow$ Statistique médicale) ou d'envoyer un courriel à ddq[at]fmh.ch.

\section{Références}

1 Sur la situation actuelle en matière de prise en charge médicale: www.bag.admin.ch/themen/berufe/13932/13933/14201/index. html?lang=fr

2 Dutoit L, Gardiol L,Künzi K. Etablissement du recensement des médecins actifs en Suisse (Obsan Dossier 28). Neuchâtel: Observatoire suisse de la santé; 2014.

3 Hostettler S, Laffranchi R, Kraft E. Taux d'occupation des médecins en Suisse. Bull Méd Suisses. 2013; 95(50):1891-5.

4 Kraft E, Napierala C. Validation des données en vue de la recherche sur la fourniture des soins. Bull Méd Suisses. 2010;91(3):64-6.

5 Médecine humaine: titres fédéraux en 2013: www.bag.admin.ch/ themen/berufe/13930/13936/14382/index.html?lang=fr

6 Les pays avec la plus forte densité médicale dans le monde en 2013 (en allemand): http://de.statista.com/statistik/daten/studie/ 232536/umfrage/laender-mit-der-hoechsten-aerztedichteweltweit/

7 Une stratégie globale pour la santé publique: www.bag.admin.ch/ gesundheit2o2o/index.html?lang=fr

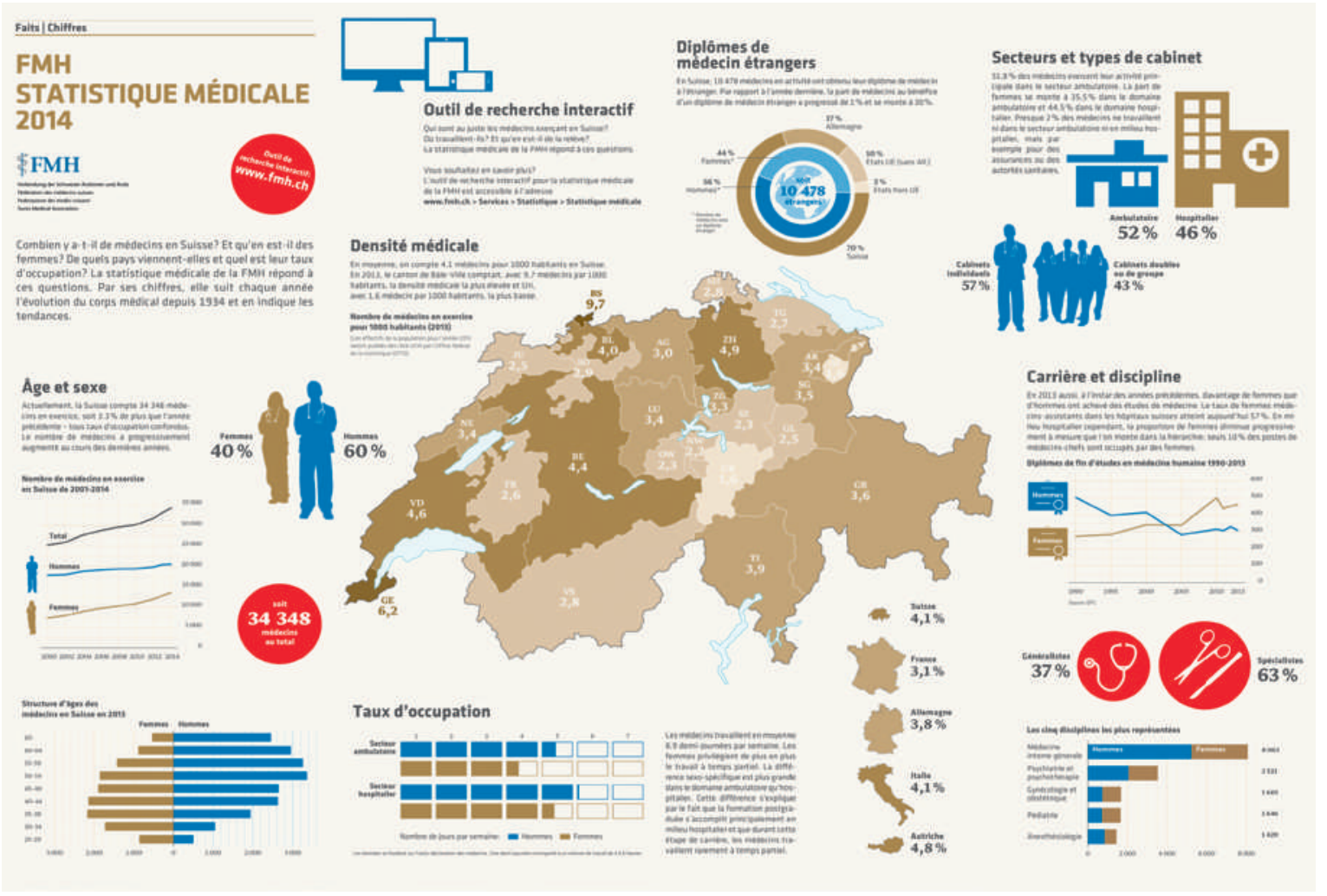




\section{Glossaire (par ordre alphabétique)}

Correspondance: FMH / division DDQ Elfenstrasse 18 CH-30oo Berne 15 Tél. 0313591111 www.fmh.ch

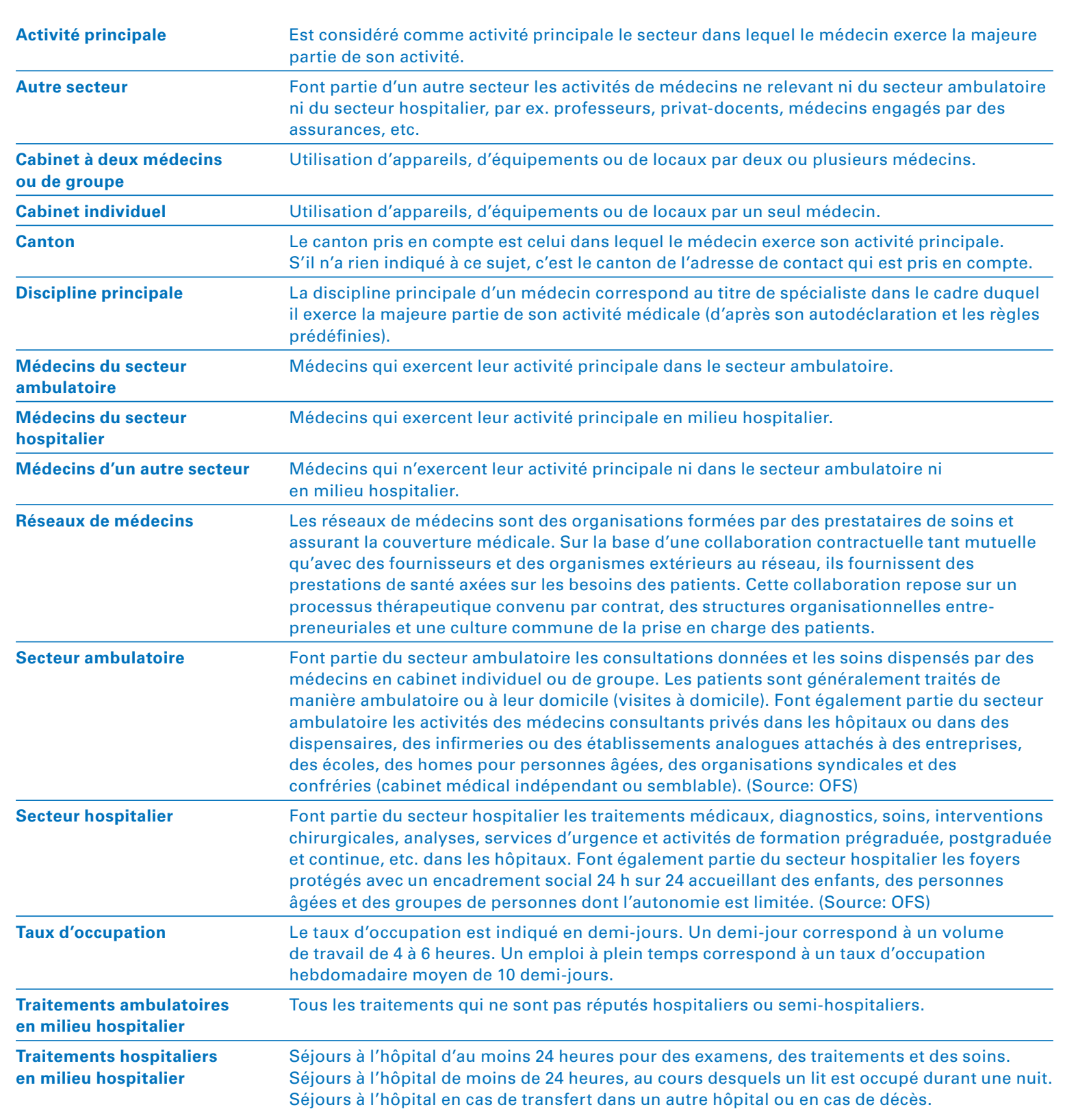

